\title{
A simple method for nicardipine hydrochloride quantification in plasma using solid-phase extraction and reversed-phase high-performance liquid chromatography
}

\author{
Catarina Marques Fernandes and Francisco José Baptista Veiga* \\ Laboratory of Pharmaceutical Technology, Faculty of Pharmacy, University of Coimbra, Coimbra, Portugal
}

Received 30 August 2002; revised 17 October 2002; accepted 21 October 2002

\begin{abstract}
A simple and sensitive reversed-phase liquid chromatography method was developed and validated for the determination of nicardipine hydrochloride (NC) in rabbit plasma. Nicardipine hydrochloride and nimodipine, used as internal standard, were initially extracted from plasma by a rapid solid-phase extraction using $\mathrm{C}_{18}$ cartridges. After extraction, nicardipine hydrochloride was separated by HPLC on a $\mathrm{C}_{18}$ column and quantified by ultraviolet detection at $254 \mathrm{~nm}$. A mixture of acetonitrile$0.02 \mathrm{M}$ sodium phosphate buffer-methanol (45:40:15) with $0.2 \%$ of triethylamine of $\mathrm{pH}$ of 6.1 was used as mobile phase. The mean $( \pm \mathrm{SD})$ extraction efficiency of $\mathrm{NC}$ was $77.56 \pm 5.4,84.23 \pm 4.32$ and $83.94 \pm 3.87 \%$ for drug concentrations of 5, $25 \mathrm{and} 100 \mathrm{ng} / \mathrm{mL}$, respectively. The method proved to be linear in the range of $5-100 \mathrm{ng} / \mathrm{mL}$ with a regression coefficient of 0.9993 . The relative standard deviations of intra- and inter-day analysis for NC in plasma were 3.26-6.52\% $(n=5)$ and 4.71-9.38\% ( $n=5)$, respectively. The differences of the mean value measured from the concentration prepared, expressed in percentages (bias percentage), were only $-5.2,0.4$ and $0.8 \%$ at NC 5, 25 and $50 \mathrm{ng} / \mathrm{mL}$, which confirmed the accuracy of the method. The analytical technique was used to determine NC plasma concentration after drug oral administration to rabbits. The results inferred that NC is rapidly absorbed in rabbits and has a short half-life $\left(t_{1 / 2}=1.34 \mathrm{~h}\right)$. Copyright (C) 2003 John Wiley \& Sons, Ltd.
\end{abstract}

KEYWORDS: Nicardipine Hydrochloride; reversed-phase HPLC; solid-phase extraction

\section{INTRODUCTION}

Nicardipine hydrochloride (NC) is a calcium-channel blocking agent, which is widely used in the management of mild to moderate hypertension, angina pectoris and cerebrovascular disease due to its potent vasodilating activity (Iwanami et al., 1979; Sorkin and Clissold, 1987; Terai et al., 1981). This drug, like other dihydropyridine derivatives, undergoes rapid absorption and extensive biotransformation in the liver, after oral administration to humans and laboratory animals, which display relatively low plasma concentrations (Higuchi and Shrobara, 1980; Sorkin and Clissold, 1987). For this reason, a sensitive and specific method is required for NC quantification in plasma samples.

Several methods for NC determination in human or laboratory animals plasma have been reported, including gas chromatography (GC) (Watari et al., 1990), GC with electron-capture detection (Higuchi et al., 1975), GCmass spectrometry (Marciniec and Kujawa, 1995),

*Correspondence to: F. J.-. B. Veiga, Faculdade de Farmácia, Universidade de Coimbra, Rua do Norte, 3000 Coimbra, Portugal. E-mail: fveiga@ci.uc.pt

Abbreviations used: NC, nicardipine hydrochloride; NM, nimodipine. combination of thin-layer chromatography with GC and mass spectrometric detection (Higuchi and Kawamura, 1981) and voltammetry (Obendorf and Stubauer, 1995). However, some of these methods are too complex and cumbersome for the routine analysis of large numbers of samples and involve unconventional, specialized and expansive equipment (Higuchi and Kawamura, 1981) and others are not sensitive enough for accurate quantification of low therapeutic concentrations of NC (Obendorf and Stubauer, 1995), which limits their application. In addition, some of these methods (eg GC techniques) are relatively non-specific because require $\mathrm{NC}$ oxidation prior to the analysis.

In recent years, high-performance liquid chromatography (HPLC) either with ultraviolet (Greiner et al., 1988; Kobayashi, 1987; Li et al., 1998; Uno et al., 1997) or electrochemical detection (Eastwood et al., 1990; Telting-Diaz et al., 1991) has overcome some of these diffuculties and has been extensively used to determine $\mathrm{NC}$ in biological samples. Nevertheless, almost all the published methods of HPLC for the measurement of NC in plasma, involve relatively laborious and tedious liquid-liquid extraction procedures prior to HPLC analysis, which limit their application for the analysis of large number of samples resulting from clinical or experimental research. The HPLC method described here 


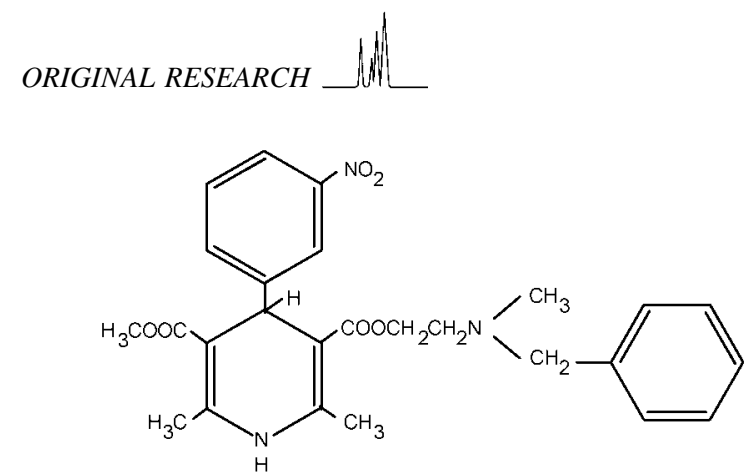

(A)<smiles>CCOC(=O)C1=C(C)NC(C)=C(C(=O)OCC)C1c1cccc([N+](=O)[O-])c1</smiles>

(B)

Figure 1. Chemical structures of nicardipine (A) and nimodipine (B).

overcomes this question by means of a rapid solid phase extraction (SPE) procedure followed by the direct injection of the extract into the chromatographic system.

A simple, rapid and precise method for the determination of NC in plasma, involving solid phase extraction for sample preparation, followed by a reversed-phase HPLC technique, was developed, optimized and validated. Further, the analytical technique was applied to characterize the pharmacokinetic profile of $\mathrm{NC}$ after oral administration to rabbits.

\section{EXPERIMENTAL}

Materials and reagents. NC (100.37\%) and nimodipine (NM) (98.7\%), used as internal standard, were purchased from Effechem SRL (Milan, Italy) and Sigma-Aldrich (Steinheim, Germany), respectively. The chemical structures of both drugs are shown in Fig. 1. Acetonitrile, methanol, ethanol and chloroform were of HPLC grade (Merck). All other reagents and chemicals were of analytical grade (Merck and Sigma-Aldrich) and water was obtained by a Millipore Elix 5 system.

The Spe-ed $\mathrm{C}_{18}$ cartridges (100 mg of sorbent amount) used for the solid phase extraction were obtained from Applied Separations (Allentown, USA). The vacuum manifold column processor for SPE and the corresponding drying attachment were purchased from Lida (Kenosha, USA).

Since NC and NM are light sensitive, almost all experiments were carried out in a darkroom under yellow light (Philips Powertone SON E27), in order to avoid photodecomposition. When this photoprotection was impossible to achieve, all samples were protected from light by wrapping the vials with aluminum foil.

Apparatus and chromatographic conditions. The HPLC system used consisted of a Hewlett Packard (Waldbronn, Germany) model 1050 quaternary solvent-delivery pump, equipped with a HP1050 multiple wavelength UV detector operated at $254 \mathrm{~nm}$ and an injector with a $20 \mu \mathrm{L}$ loop.

The reversed-phase chromatographic separations were carried out on a stainless-steel column $(250 \times 4 \mathrm{~mm}$ i.d. $)$ packed with Licrospher ${ }^{\mathbb{R}} 100 \mathrm{RP}_{18}$ (mean particle size $5 \mu \mathrm{m}$, Merck) maintained at $30^{\circ} \mathrm{C}$. A guard column similarly packed (Licrospher ${ }^{\mathbb{R}} 100$ $\mathrm{RP}_{18}, 5 \mu \mathrm{m}$, Merck) was used before the analytical column.
A mixture of acetonitrile-aqueous $0.02 \mathrm{M}$ sodium phosphate buffer-methanol $(45: 40: 15, \mathrm{v} / \mathrm{v})$ containing $0.2 \%(\mathrm{v} / \mathrm{v})$ of triethylamine with $\mathrm{pH}$ of 6.1 was used as mobile phase. In previous studies, various mobile phases were tested with different organic solvents contents and $\mathrm{pH}$. The $\mathrm{pH}$ of the mobile phase was adjusted using either $1 \%(\mathrm{v} / \mathrm{v})$ phosphoric acid and $0.1 \mathrm{~N}$ sodium hydroxide solution. The final eluting system was selected in terms of optimum resolution and peak shapes of NC with a short retention time. The HPLC eluent was filtered through $0.45 \mu \mathrm{m}$ membrane filters (PVDF, Tracer) and was degassed by sonication or with a vigorous stream of helium. The mobile phase was delivered isocratically at a flow rate of $1.2 \mathrm{~mL} / \mathrm{min}$.

Chromatographic peak heights were measured using a Hewlett Packard model 3396A recording integrator.

Preparation of standard solutions. Stock standard solutions of $\mathrm{NC}$ and NM were prepared in absolute ethanol at a concentration of 3.4 and $2.5 \mu \mathrm{g} / \mathrm{mL}$, respectively, by diluting the corresponding $1 \mathrm{mg} / \mathrm{mL}$ ethanolic solution. All the stock solutions were kept at $4^{\circ} \mathrm{C}$ in glass wrapped with aluminum foil.

For each validation run, a $100 \mathrm{ng} / \mathrm{mL}$ solution of $\mathrm{NC}$ in plasma was freshly prepared by appropriate dilution of the $3.4 \mu \mathrm{g} / \mathrm{mL}$ ethanolic solution with $\mathrm{NC}$-free rabbit plasma.

Procedure for sample preparation. The internal standard ( $20 \mu \mathrm{L}$ of the $2.5 \mu \mathrm{g} / \mathrm{mL}$ ethanolic solution) and $30 \mu \mathrm{L}$ of $0.1 \mathrm{M}$ $\mathrm{NaOH}$ solution were added to $800 \mu \mathrm{L}$ of plasma sample. After $30 \mathrm{~s}$ of vortex mixing, the mixture was applied to a Spe-ed $\mathrm{C}_{18}$ cartridge that had previously been conditioned with $3 \mathrm{~mL}$ of ethanol and $3 \mathrm{~mL}$ of water. After the complete aspiration of the sample solution through the cartridge, the extraction column was air dried under vacuum for $2 \mathrm{~min}$ with a SPE column processor. The cartridge was then washed with $3 \mathrm{~mL}$ of water and the residual water was removed from the extraction column under vacuum for $5 \mathrm{~min}$. The desired fraction was further eluted with four portions of $200 \mu \mathrm{L}$ of chloroform. Among various organic solvents tested for the elution procedure, chloroform proved to be the most efficient and selective. The eluate was evaporated to dryness under a stream of nitrogen in vacuum and at room temperature. The residue was dissolved in $80 \mu \mathrm{L}$ of the HPLC eluent. After 1 min of vortex mixing, $50 \mu \mathrm{L}$ of the sample solution were injected into the HPLC system for analysis.

Validation of the method. The linearity of the method was tested between 5 and $100 \mathrm{ng} / \mathrm{mL}$. The spiked plasma standards 
containing $5,10,25,50$ and $100 \mathrm{ng} / \mathrm{mL}$ of $\mathrm{NC}$ were prepared by dilution from the $100 \mathrm{ng} / \mathrm{mL}$ plasma solution freshly prepared. The different spiked plasma samples were processed according to the extraction procedure described above. A calibration curve was constructed by plotting the NC/NM peak-height ratios against the corresponding NC concentrations in plasma. The plot was subject to a linear regression analysis and the unknown NC concentrations were determined from the regression equation. The data obtained were used for the subsequent study of the validation parameters.

NC standard plasma solutions covering different concentration levels $(5,25$ and $100 \mathrm{ng} / \mathrm{mL})$, prepared by diluting the $100 \mathrm{ng} / \mathrm{mL}$ plasma solution, were used to determine the precision and the accuracy of the method. For the inter-day assay the standards were prepared over a 5-day period, while the intra-day assay was performed for 1 day by analyzing each concentration five times. The NC standard plasma solutions were also processed by the extraction technique previously reported.

To establish the extraction efficiency, NC plasma standard solutions were prepared by adding aliquots of the $100 \mathrm{ng} / \mathrm{mL}$ plasma solution to drug-free plasma, at three different concentration levels $(5,25$ and $100 \mathrm{ng} / \mathrm{mL})$. The recovery percentage of $\mathrm{NC}$ was determined by comparing the drug/internal standard peak height ratios after extraction of the spiked samples with the corresponding ratios obtained after direct injection of nonextracted NC standard solutions in mobile phase, at same concentrations. The direct injection analysis was considered as the $100 \%$ of recovery. The mean extraction efficiency was obtained by assaying five replicates for each concentration of NC.

In a preliminary study performed during a period of 4 months, $\mathrm{NC}$ plasma samples proved to be stable when stored at -80 or $-25^{\circ} \mathrm{C}$

Drug administration and sampling. Six male rabbits with an average weight of $4 \mathrm{~kg}$ were kept under fasting conditions for at least $12 \mathrm{~h}$ before dosing. $\mathrm{NC}(15 \mathrm{mg} / \mathrm{kg})$ was orally administered, in gelatin capsules, to six animals. Blood samples $(1.5 \mathrm{~mL})$ were collected in tubes with EDTA, prior to NC administration and at $0.5,1,2,3,4,6,8$ and $10 \mathrm{~h}$ after the drug administration. Plasma samples were separated by centrifugation at $3000 \mathrm{rpm}$ for $10 \mathrm{~min}$ and stored at $-80{ }^{\circ} \mathrm{C}$ until analysis. Baseline plasma samples obtained prior to $\mathrm{NC}$ administration at time 0 served as the blank control for each animal.

\section{RESULTS AND DISCUSSION}

\section{Procedure for sample preparation}

According to preliminary solubility studies, chloroform, acetic acid, ethanol, methanol and acetonitrile (with or without $1 \%$ of triethylamine) were tested as elution solvents in the SPE of NC and NM. Some of these solvents caused a poor NC extraction (eg ethanol) and others promoted the coextraction of various constituents of plasma or components of the cartridges (eg methanol and triethylamine), interfering with the HPLC analysis of NC. These results were not in agreement with studies previously reported, which used methanol (Uno et al., 1997) and methanol with $1 \%$ of

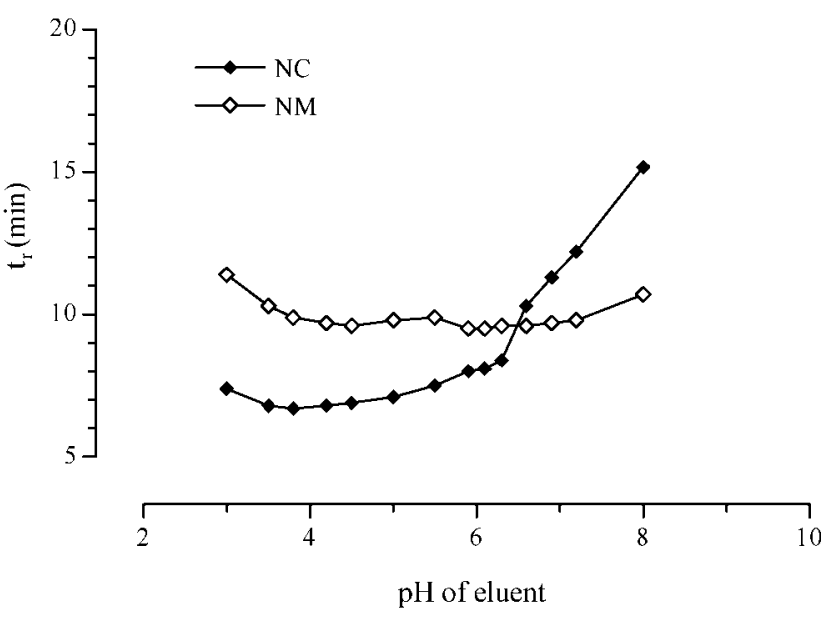

Figure 2. Influence of eluent $\mathrm{pH}$ on the retention time of NC and NM.

triethylamine (Meng et al., 1998) as elution solvents. In the present study, chloroform proved to be the most selective and efficient eluting solvent since it greatly reduced the interfering peaks in the plasma chromatograms and gave the highest recovery rates for NC and NM. In addition, to achieve higher extraction efficiency, $\mathrm{NaOH}$ should be added to the initial plasma sample, to assure that the whole $\mathrm{NC}$ is in the molecular form, by adjusting the $\mathrm{pH}$ above the drug $\mathrm{p} K_{\mathrm{a}}$ $\left(\mathrm{p} K_{\mathrm{a}}=7.2\right)$. This procedure will reduce the analyte solubility in the sample solution and improve the retention by the sorbent bed of the cartridge.

\section{Optimization of the chromatographic method}

Although the UV absorption maximum of NC and NM was at $238 \mathrm{~nm}$, in this study the absorption at $254 \mathrm{~nm}$ was used for quantification. The use of the absorption at $254 \mathrm{~nm}$ reduces the theoretical sensitivity to some extent, but has the enormous advantage of providing cleaner chromatograms for plasma samples.

The separation of the analytes, peak shapes and retention times were optimized by modifying the mobile phase constitution and the flow rate until attaining a good resolution in a relatively short run time for each injection (12 min).

The effect of the mobile phase composition on the chromatographic separation was firstly investigated. The results showed that NC and NM retention times were obviously prolonged with decreasing the acetonitrile and/ or methanol content or increasing the buffer content. However, the responses of peak height changed only slight with varying composition of the mobile phase. The presence of methanol was found to be necessary to ensure a good separation between NC, NM and plasma interfering peaks. Further, the experimental results showed that the addition of triethylamine in the mobile 

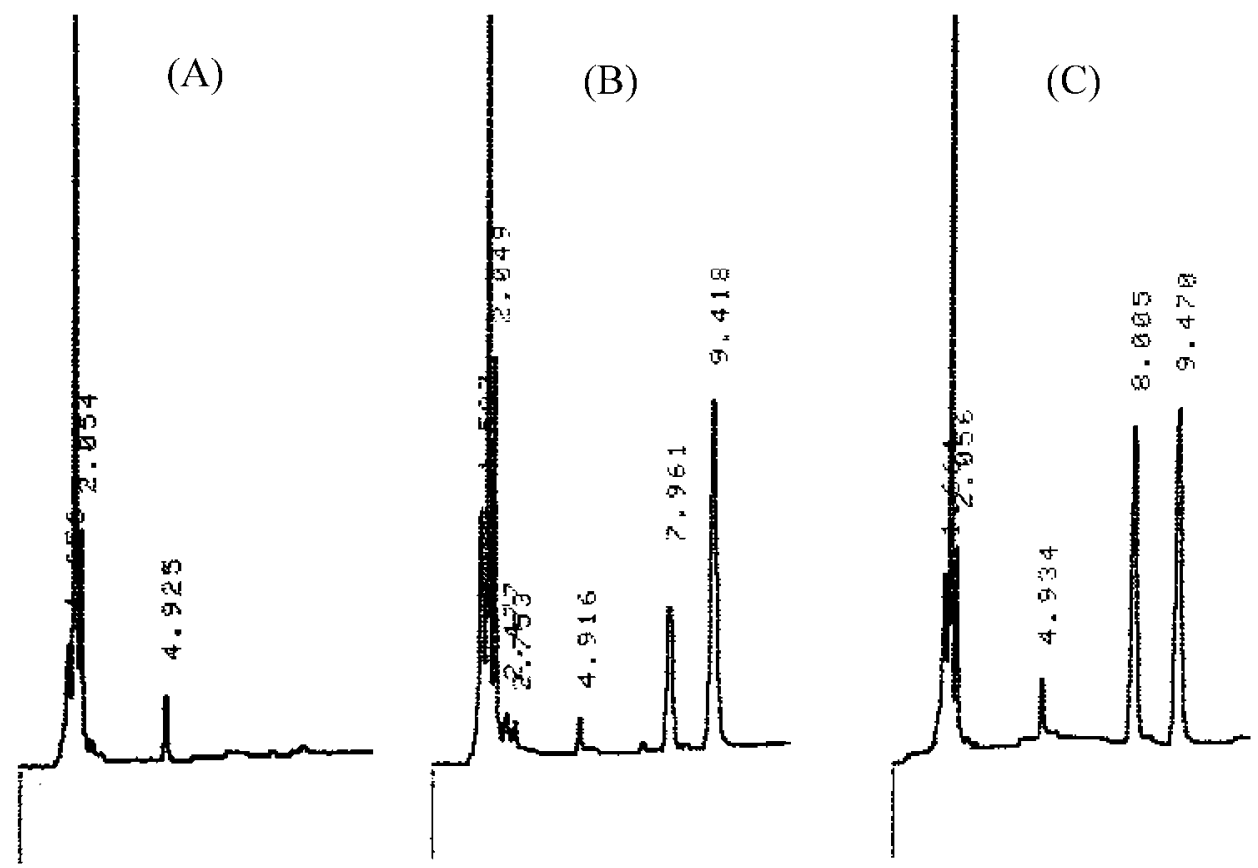

Figure 3. Typical chromatograms of extracted rabbit plasma: (A) rabbit drug-free plasma; (B) rabbit plasma spiked with $\mathrm{NC}\left(t_{\mathrm{r}}=7.961 \mathrm{~min}\right)$ and $\mathrm{NM}\left(t_{\mathrm{r}}=9.418 \mathrm{~min}\right)$ and $(\mathrm{C})$ plasma sample from an animal treated with $15 \mathrm{mg} / \mathrm{kg}$ of NC. $t_{\mathrm{r}}=$ retention time.

phase increased the selectivity and sensitivity of NC, because it changed the retention time and sharpened the drug peak. To maintain the elution order (NC before its internal standard) and the quality of the separation, it was also necessary to adjust the $\mathrm{pH}$ of the mobile phase. As can be seen in Fig. 2, the chromatographic behavior of $\mathrm{NM}$, a neutral substance, was not strongly affected by the variation of $\mathrm{pH}$ of the HPLC eluent. Nevertheless, this factor played an important role in the retention time of $\mathrm{NC}$, a basic drug. At low values of $\mathrm{pH}, \mathrm{NC}$ eluted at shorter time, because the high degree of protonation of its tertiary amine function hinders the interactions with the lipophilic stationary phase. An optimal $\mathrm{pH}$ of 6.1 was chosen for selecting a proper retention time value that produced no interfering peaks near the specific peak of $\mathrm{NC}$ and because is at least $1 \mathrm{pH}$ unit different from the $\mathrm{NC}$ pKa, which shifts the equilibrium so that $99 \%$ of $\mathrm{NC}$ will be in one form. Analytes may sometimes appear as broad or tailing peaks when the mobile phase $\mathrm{pH}$ is at, or near, their $\mathrm{p} K_{\mathrm{a}}$ values.

From these preliminary studies, we decided to use a mixture of acetonitrile-aqueous $0.02 \mathrm{M}$ sodium phosphate buffer-methanol $(45: 40: 15, \mathrm{v} / \mathrm{v})$ containing $0.2 \%$ $(\mathrm{v} / \mathrm{v})$ of triethylamine with $\mathrm{pH}$ of 6.1 as mobile phase at a flow rate of $1.2 \mathrm{~mL} / \mathrm{min}$.

Typical chromatograms of a standard mixture of NC and NM in rabbit plasma samples, is shown in Fig. 3. Under the chromatographic conditions described above, the observed mean retention times for NC and NM were
8.0 and $9.5 \mathrm{~min}$, respectively. The lack of response in blank plasma was demonstrated.

\section{Validation of the method}

As it can be seen in Fig. 3, a good separation of NC with minimal interference with internal standard or with endogenous components of plasma was obtained by the present extraction and chromatographic techniques. It can be concluded that the proposed method is selective for NC.

The calibration curve of NC/NM peak-height vs NC concentration in plasma was linear in the concentration range of $5-100 \mathrm{ng} / \mathrm{mL}$ with a correlation coefficient of $0.9993(n=5)$, which proved excellent linearity in this concentration range.

The accuracy and precision (intra-day precision or repeatability and inter-day or intermediate precision) of this method, evaluated by assaying five spiked plasma samples containing different concentrations of NC, are summarized in Table 1. The differences of the mean value measured from the concentration prepared, expressed in percentages (bias percentage), were only $-5.2,0.4$ and $0.8 \%$ at 5,25 and $50 \mathrm{ng} / \mathrm{mL}$, which confirmed the accuracy of the method. The range of percentage of relative standard deviation (RSD) was $3.26-6.52 \%$ and $4.71-9.38 \%$ for within-day and between-day analyses, respectively. The RSD values 
Table 1. Accuracy and precision of the method for the determination of $\mathrm{NC}$ in rabbit plasma, expressed as bias (\%) and relative standard deviation $(\mathrm{RSD})$, respectively $(n=5)$

\begin{tabular}{rccr}
\hline Concentration prepared $(\mathrm{ng} / \mathrm{mL})$ & Accuracy Bias $(\%)$ & Intra-day precision RSD $(\%)$ & Inter-day precision RSD $(\%)$ \\
\hline 5 & -5.20 & 6.52 & 9.38 \\
25 & 0.43 & 3.26 & 5.32 \\
100 & 0.78 & 4.07 & 4.71 \\
\hline
\end{tabular}

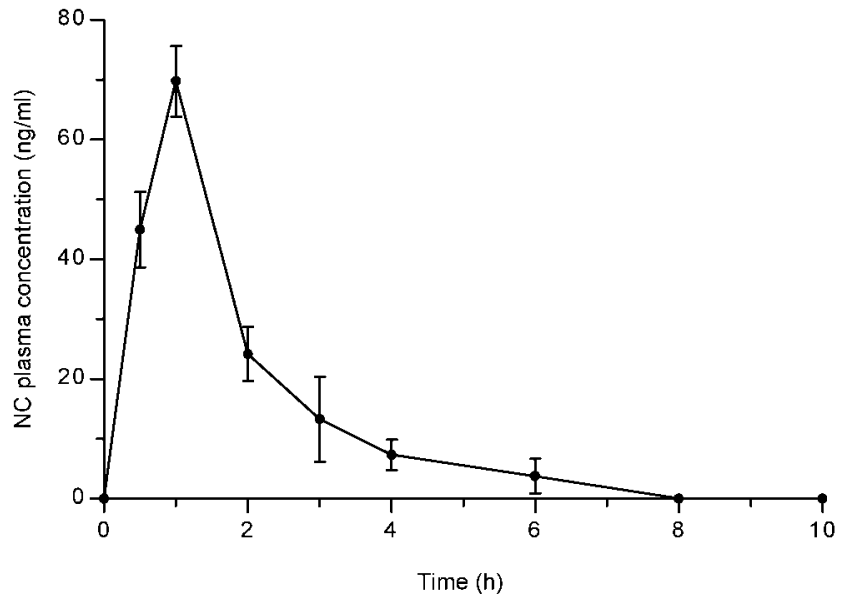

Figure 4. Mean $( \pm$ SEM) drug plasma concentrations after oral administration of a single dose of $15 \mathrm{mg} / \mathrm{kg}$ of $\mathrm{NC}$ to six rabbits.

obtained allow us to conclude that the method has acceptable precision, although a slightly greater variation has been found at $5 \mathrm{ng} / \mathrm{mL}$.

The mean $( \pm \mathrm{SD})$ extraction efficiency for $\mathrm{NC}$ from plasma was found to be consistent over the evaluated concentrations: $77.56 \pm 5.4, \quad 84.23 \pm 4.32$ and 83.94 $\pm 3.87 \%$, for the drug concentrations 5,25 and $100 \mathrm{ng} /$ $\mathrm{mL}$, respectively, when compared with non-extracted samples containing NC in the same concentration $(n=5)$. The extraction recovery of the internal standard from plasma was $86.6 \pm 5.9(n=5)$.

\section{Application to pharmacokinetic studies in rabbits}

Representative plasma concentration-time profile of NC after administration of $15 \mathrm{mg} / \mathrm{kg}$ of drug to rabbits is illustrated in Fig. 4, as evidence of the usefulness of this method. The mean pharmacokinetic parameters were calculated for the six animals. The absorption of $\mathrm{NC}$ in rabbits was very rapid, with a time to reach the maximum plasma level $(69.5 \mathrm{ng} / \mathrm{mL})$ of $1 \mathrm{~h}$. NC presented a short elimination half-life $(1.34 \mathrm{~h})$ and the area under the $\mathrm{NC}$ plasma concentration-time curve up to $10 \mathrm{~h}\left(\mathrm{AUC}_{0-10 \mathrm{~h}}\right)$ was estimated as $132 \mathrm{ng} \mathrm{h} / \mathrm{mL}$.

\section{CONCLUSION}

The present analytical technique consisted of a simple solid-phase extraction procedure and a subsequent reversed-phase HPLC analysis with UV detection, which could be easy reproduced in conventional equipments available in most laboratories. The present method proved to be, according to the validation parameters, selective precise and accurate and also simple, useful and appropriate for studying the pharmacokinetic profile of NC administered to laboratory animals, such as rabbits.

\section{REFERENCES}

Eastwood RJ, Galustian C, Bhamra RK and Holt DW. Highperformance liquid chromatographic method for the measurement of nicardipine in plasma or serum. Journal of Chromatgraphy: Biomedical Applications, 1990; 530: 463.

Greiner PO, Angignard D and Cahn J. High performance liquid chromatography of a new 1,4-dihydropyridine: applications to pharmacokinetic study in dogs. Journal of Pharmaceutical Science, 1988; 77: 387.

Higuchi $S$ and Kawamura J. Specific determination of plasma nicardipine levels by gas chromatography-mass spectroscopy. Journal of Chromatgraphy, 1981; 223: 341 .

Higuchi S and Shrobara Y. Comparative pharmacokinetics of nicardipine hydrochloride, a new vasodilator in various species. Xenobiotica, 1980; 10: 447.

Higuchi S, Sasaki H and Sado T. Determination of a new cerebral vasodilator 2,6-dimethyl-4-(3-nitrophenyl)-1,4-dihydropyridine3,5dicarboxylic acid 3-[2-( $N$-benzyl- $N$-methylamino)]-ethyl ester hydrochloride (YC-93) in plasma by electron capture gas chromatography. Journal of Chromatgraphy, 1975; 110: 301.

Iwanami M, Shibanuma M, Fujimoto M, Kawai R, Tamazawa K, Takenaka T, Takahashi K and Murakami M. Synthesis of new water-soluble dihyropyridine vasodilators. Chemical Pharmaceutical Bulletin, 1979; 27: 1426.

Kobayashi S. Simple method for the determination of nicardipine in plasma using high-performance liquid chromatography. Journal of Chromatgraphy: Biomedical Applications, 1987; 420: 439.

Li K, Zhang X, Yuan Y and Zhao F. A high-performance liquid chromatographic method for the determination of nicardipine in plasma and its application to pharmacokinetics in humans. Biomedical Chromatography, 1998; 12: 326.

Marciniec B and Kujawa E. Qualitative-quantitative GC-MS analysis of some dihydropyridine derivatives. Chemical Analysis, 1995; 40: 511 .

Meng QC, Cheung AT, Guvakov D, Weiss SJ, Savino JS, Salgo IS and Marshall BE. Extraction and quantification of nicardipine in human plasma. Journal of Chromatgraphy B, 1998; 718: 121.

Obendorf D and Stubauer G. Adsorptive stripping voltammetry of nicardipine at a HMDE: determination of trace levels nicardipine in blood and urine. Journal of Pharmaceutical Biomedical Analysis, 1995; 13: 1339.

Sorkin E and Clissold S. Nicardipine. A review of its pharmacody- 
namic and pharmacokinetic properties and therapeutic efficacy, in the treatment of angina pectoris, hypertension and related cardiovascular disorders. Drugs, 1987; 33: 296.

Telting-Diaz M, Kelly MT, Hua C and Smyth MR. High-performance liquid chromatographic determination of nifedipine, nicardipine and pindolol using a carbon fibre flow-through amperometric detector. Journal of Pharmaceutical Biomedical Analysis, 1991; 9: 889.

Terai M, Takenaka $\mathrm{T}$ and Maeno $\mathrm{H}$. Inhibition of calcium influx in rabbit aorta by nicardipine hydrochloride (YC-93). Biochemical Pharmacology, 1981; 30: 375.
Uno T, Ohkubo T and Sugawara K. Enantioselective high-performance liquid chromatographic determination of nicardipine in human plasma. Journal of Chromatgraphy B, 1997; 698: 181.

Watari N, Mizumura J and Higuchi S. Simultaneous microdetermination of nicardipine and its pyridine metabolite in body fluids by capillary column gas chromatography with electroncapture detection. Journal of Chromatgraphy: Biomedical Applications, 1990; 530: 438 